\title{
Hyers-Ulam-Rassias stability of non-linear delay differential equations
}

\author{
Akbar Zada $^{\mathrm{a}}$, Shah Faisal ${ }^{\mathrm{a}}$, Yongjin Li $\mathrm{i}^{\mathrm{b}, *}$ \\ ${ }^{a}$ Department of Mathematics, University of Peshawar, Peshawar 25000, Pakistan. \\ ${ }^{b}$ Department of Mathematics, Sun Yat-Sen University, Guangzhou, 510275, P. R. China.
}

Communicated by J. Brzdek

\begin{abstract}
In this paper, we prove the Hyers-Ulam-Rassias stability and Hyers-Ulam stability of delay differential equation of the form

$$
y^{(n)}=F\left(t,\left\{y^{(i)}(t)\right\}_{i=0}^{n-1},\left\{y^{(i)}(t-\lambda)\right\}_{i=0}^{n-1}\right),
$$

with Lipschitz condition by using fixed point approach. The results of the paper generalize most of the results concerning the stability of delay differential equations in the existing literature. (C)2017 All rights reserved.
\end{abstract}

Keywords: Fixed point theorem, Hyers-Ulam stability, Hyers-Ulam-Rassias stability, non-linear delay differential equations. 2010 MSC: 35B35.

\section{Introduction}

The issue of stability of functional equations has appeared in connection with a question that Ulam asked in 1940: "when an approximate solution of a functional equation can be approximated by one of exact solutions of the given equation?"(for more details see [23]). Hyers [8], by using direct method, brilliantly gave a partial answer for the case of the additive Cauchy functional equation for mappings between Banach spaces. This result was then improved by Aoki [1] and Rassias [21], who weakened the condition for the bound of the norm of Cauchy difference.

The stability phenomenons proved in [8] and [21] were named Hyers-Ulam and Hyers-Ulam-Rassias stability due to the high influence of Hyers and Rassias on this area of research. For discussion on terminology concerning that type of stability we recommend [17].

As far as we know, works by Obloza $[18,19]$ were among the first contributions dealing with the Hyers-Ulam stability of differential equations. Since then, Hyers-Ulam stability and Hyers-Ulam-Rassias stability of various classes of differential equations and differential operators were explored by using a wide spectrum of approaches; see $[2,4,5,9,10,12,14,16,24-27]$ and the references cited there.

Obloza in [19] established the connections between Hyers-Ulam and Lyapunov stability of ordinary differential equations. Recently, Li and Zada in [15] studied the connections between Hyers-Ulam and uniform exponential stability of discrete evolution families of bounded linear operators over Banach spaces.

\footnotetext{
*Corresponding author

Email addresses: zadababo@yahoo.com (Akbar Zada), shahfaisal8763@gmail.com (Shah Faisal), stslyj@mail.sysu.edu.cn (Yongjin Li)
}

doi:10.22436/jnsa.010.02.15

Received 2016-01-18 
Among the differential equations, a delay differential equation (DDE) is a differential equation where the time derivatives at the current time depend on the solution and possibly its derivatives at previous times. Delay differential equations differ from ordinary differential equations in that the derivative at any time depends on the solution (and in the case of neutral equations on the derivative) at prior times.

In recent years, Hyers-Ulam stability and Hyers-Ulam-Rassias stability of delay differential equations have attracted interest of researchers; see, for instance, $[6,7,11,20,22]$. One of the main reasons for this lies in the fact that, as pointed out by Kuang [13], delay differential equations arise in a number of applied problems in natural sciences and engineering. For details on applications we recommend the monograph by Kuang [13]. Note that the results reported in [6, 7, 11, 20, 22] are concerned with stability of several classes of first-order non-linear and linear delay differential equations. Thereinto, Huang et al. [7] recently proved the Hyers-Ulam stability for first non-linear delay differential equations on a compact interval. So far, to the best of our knowledge, Ulam's type stability results of higher-order non-linear delay ordinary differential equations have not been studied.

In this paper we propose study of both Hyers-Ulam and Hyers-Ulam-Rassias stability for the higher order non-linear delay differential equations of the form

$$
\left\{\begin{array}{l}
y^{(n)}(t)=F\left(t,\left\{y^{(i)}(t)\right\}_{i=0}^{n-1},\left\{y^{(i)}(t-\lambda)\right\}_{i=0}^{n-1}\right), \quad t \in\left[t_{0}, T\right], \\
y^{(i)}(t)=\Upsilon^{(i)}(t), \quad i=0,1, \cdots, n-1, \quad t \in\left[t_{0}-\lambda, t_{0}\right],
\end{array}\right.
$$

where $t_{0}<T, 0<\lambda$ and $\Upsilon:\left[t_{0}-\lambda, t_{0}\right] \rightarrow R$ is $n-1$ times continuously differentiable function.

\section{Notations and preliminaries}

In this section we introduce notations, definitions, and preliminary facts which are used throughout this paper.

Throughout the paper $\mathrm{D}^{\mathrm{n}}\left[\mathrm{t}_{0}-\lambda, T\right]$ represents the class of all real-valued functions which are $n$ times continuously differentiable on $\left[t_{0}-\lambda, T\right]$. The Hyers-Ulam and Hyers-Ulam-Rassias stability for the case of problem (1.1) can be defined as follows.

Definition 2.1. The initial value problem (1.1) is said to have the Hyers-Ulam stability on $\left[t_{0}-\lambda, T\right]$, if and only if for every function $y \in \mathrm{D}^{\mathfrak{n}}\left[\mathrm{t}_{0}-\lambda, T\right]$ and $\epsilon \geqslant 0$ such that

$$
\left\{\begin{array}{c}
\left|y^{(n)}(t)-F\left(t,\left\{y^{(i)}(t)\right\}_{i=0}^{n-1},\left\{y^{(i)}(t-\lambda)\right\}_{i=0}^{n-1}\right)\right| \leqslant \epsilon, \quad t \in\left[t_{0}, T\right], \\
\left|y^{(i)}(t)-\Upsilon^{(i)}(t)\right| \leqslant \epsilon, \quad i=0,1, \cdots, n-1, \quad t \in\left[t_{0}-\lambda, t_{0}\right],
\end{array}\right.
$$

there is a function $y_{0} \in D^{\mathfrak{n}}\left[t_{0}-\lambda, T\right]$ with

$$
\left\{\begin{array}{l}
y_{0}^{(n)}(t)=F\left(t,\left\{y_{0}^{(i)}(t)\right\}_{i=0}^{n-1},\left\{y_{0}^{(i)}(t-\lambda)\right\}_{i=0}^{n-1}\right), \quad t \in\left[t_{0}, T\right], \\
y_{0}^{(i)}(t)=\Upsilon^{(i)}(t), \quad i=0,1, \cdots, n-1, \quad t \in\left[t_{0}-\lambda, t_{0}\right],
\end{array}\right.
$$

and

$$
\left|y_{0}(t)-y(t)\right|<K(\epsilon), \quad \forall t \in\left[t_{0}-\lambda, T\right],
$$

where $\mathrm{K}(\epsilon) \rightarrow 0$ as $\epsilon \rightarrow 0$.

If the above statement is also true when we replace $K(\epsilon)$ and $\epsilon$ by $\Phi(t)$ and $\ell(t)$, in turn, where $\ell, \Phi:\left[t_{0}-\lambda, T\right] \rightarrow(0, \infty)$ are functions not depending on $y$ and $y_{0}$ explicitly, then we say that the corresponding differential equation has the generalized Hyers-Ulam stability (or the Hyers-Ulam-Rassias stability).

Definition 2.2. A function $d: X \times X \rightarrow[0, \infty]$, where $X$ is any non-empty set, is called generalized metric on $X$, if and only if for all $x, y, z \in X$ the following hold: 
(p1) $d(x, y)=d(y, x)$;

(p2) $d(x, y)=0$, if and only if $x=y$;

(p3) $d(x, y) \leqslant d(x, z)+d(z, y)$

It is noteworthy that the only difference between the ordinary metric and the generalized metric is that the latter one can attain $\infty$ in its range.

We will use the following theorem in deriving our main results. For proof see [3].

Theorem 2.3. Let $(\mathrm{X}, \mathrm{d})$ be a generalized complete metric space and $\mathfrak{R}: \mathrm{X} \rightarrow \mathrm{X}$ be a strictly contractive operator with the Lipschitz constant $\mathrm{M}<1$. If there is a nonnegative integer $\mathrm{m}$ such that $\mathrm{d}\left(\mathfrak{R}^{\mathrm{m}+1} \chi, \mathfrak{R}^{\mathrm{m}} \mathrm{x}\right)<\infty$ for some $x \in X$, then the following are true:

(a) The sequence $\left\{\Re^{n} x\right\}$ converges to a fixed point $x_{0}$ of $\Re$ in $X$;

(b) $x_{0}$ is the unique fixed point of $\mathfrak{R}$ in

$$
X^{*}=\left\{y \in X: d\left(y, \Re^{m} x\right)<\infty\right\}
$$

(c) if $y \in X^{*}$, then

$$
d\left(y, x_{0}\right) \leqslant \frac{1}{1-M} d(\Re y, y) .
$$

Remark 2.4. The conclusion of this theorem, speaking in general terms, asserts that either all consecutive pairs of the sequence of successive approximations are infinitely far apart, or the sequence of successive approximations, with initial element converges to a fixed point of $\mathfrak{R}$.

\section{Hyers-Ulam-Rassias stability}

In this section we investigate the generalized Hyers-Ulam stability of equation (1.1).

Theorem 3.1. Let $\mathrm{K}, \mathrm{L}_{1}, \mathrm{~L}_{2}, \mathrm{~L}_{3}, \cdots, \mathrm{L}_{2 n}$ are positive constants such that $0<\mathrm{K} \sum_{i=1}^{2 n} \mathrm{~L}_{\mathfrak{i}}<1$. Assume that:

(a) $\mathrm{F}:\left[\mathrm{t}_{0}, \mathrm{~T}\right] \times \mathrm{R}^{2 \mathrm{n}} \rightarrow \mathrm{R}$ is a continuous bounded function satisfying the Lipschitz condition

$$
\left|F\left(t, x_{1}, x_{3}, \cdots, x_{2 n}\right)-F\left(t, y_{1}, y_{2}, \cdots, y_{2 n}\right)\right|<\sum_{i=1}^{2 n} L_{i}\left|x_{i}-y_{i}\right|,
$$

for all $\mathrm{t} \in\left[\mathrm{t}_{0}, \mathrm{~T}\right]$ and $\mathrm{x}_{\mathrm{i}}, \mathrm{y}_{\mathrm{i}} \in \mathrm{R}$, where $\mathrm{i}=1,2,3, \cdots, 2 \mathrm{n}$;

(b) there is a continuous function $\ell:\left[\mathrm{t}_{0}-\lambda, \mathrm{T}\right] \rightarrow(0, \infty)$ with

$$
\left|\int_{\mathrm{t}_{0}}^{\mathrm{t}} \cdots \int_{\mathrm{t}_{0}}^{v_{3}} \int_{\mathrm{t}_{0}}^{v_{2}} \int_{\mathrm{t}_{0}}^{v_{1}} \ell(v) \mathrm{d} v \mathrm{~d} v_{1} \mathrm{~d} v_{2} \mathrm{~d} v_{3} \cdots \mathrm{d} v_{\mathrm{n}-1-\mathrm{i}}\right|<\mathrm{K} \ell(\mathrm{t})
$$

for all $\mathrm{t} \in\left[\mathrm{t}_{0}, \mathrm{~T}\right]$ and $\mathrm{i}=1,2, \cdots, \mathrm{n}$.

Then for every function $\mathrm{y} \in \mathrm{D}^{\mathrm{n}}\left[\mathrm{t}_{0}-\lambda, \mathrm{T}\right]$ satisfying

$$
\left\{\begin{array}{l}
\left|y^{(n)}(t)-F\left(t,\left\{y^{(i)}(t)\right\}_{i=0}^{n-1},\left\{y^{(i)}(t-\lambda)\right\}_{i=0}^{n-1}\right)\right| \leqslant \ell(t), \quad t \in\left[t_{0}, T\right], \\
\left|y^{(i)}(t)-\Upsilon^{(i)}(t)\right| \leqslant \ell(t), \quad i=0,1, \cdots, n-1, \quad t \in\left[t_{0}-\lambda, t_{0}\right],
\end{array}\right.
$$

there is a unique function $\mathrm{u} \in \mathrm{D}^{\mathfrak{n}}\left[\mathrm{t}_{0}-\lambda, \mathrm{T}\right]$ with

$$
\left\{\begin{array}{l}
u^{(n)}(t)=F\left(t,\left\{u^{(i)}(t)\right\}_{i=0}^{n-1},\left\{u^{(i)}(t-\lambda)\right\}_{i=0}^{n-1}\right), \quad t \in\left[t_{0}, T\right] \\
u^{(i)}(t)=\Upsilon^{(i)}(t), \quad i=0,1, \cdots, n-1, \quad t \in\left[t_{0}-\lambda, t_{0}\right]
\end{array}\right.
$$

and

$$
|y(t)-u(t)| \leqslant \frac{K}{1-K \sum_{i=1}^{2 n} L_{i}} \ell(t), \quad \forall t \in\left[t_{0}-\lambda, T\right] .
$$


Proof. Define a metric on $\mathrm{D}^{\mathrm{n}}\left[\mathrm{t}_{0}-\lambda, \mathrm{T}\right]$ by

$$
d\left(y_{1}, y_{2}\right)=\inf \left\{C \in[0, \infty]: \max _{0 \leqslant i \leqslant n}\left|y_{1}^{(i)}(t)-y_{2}^{(i)}(t)\right| \leqslant C \ell(t), \quad \forall t \in\left[t_{0}-\lambda, T\right]\right\} .
$$

It is very easy to show $\left(D^{n}\left[t_{0}-\lambda, T\right], d\right)$ is metric space. We here only prove that the space is a complete metric space. For this, let $\left\{g_{k}(t)\right\}$ be a Cauchy sequence in $D^{\mathfrak{n}}\left[t_{0}-\lambda, T\right]$. Then for every $\epsilon>0$, there can be found a natural number $N_{\epsilon}$ such that

$$
\forall \mathrm{t} \in\left[\mathrm{t}_{0}-\lambda, T\right] \text { and } n, m \geqslant N_{\epsilon} \Rightarrow d\left(g_{n}(t), g_{m}(t)\right)<\epsilon .
$$

From the definition of the metric we can write

$$
\forall \mathrm{t} \in\left[\mathrm{t}_{0}-\lambda, T\right] \text { and } n, m \geqslant N_{\epsilon} \Rightarrow \max _{0 \leqslant i \leqslant n}\left|g_{n}^{(i)}(t)-g_{m}^{(i)}(t)\right|<\epsilon \ell(t) .
$$

For every fixe value of $t$ the sequence $\left\{g_{k}^{(i)}(t)\right\}$, for each $i=0,1,2, \ldots, n$, is a Cauchy sequence in $R$, but since $R$ is complete so for each $i=0,1,2, \cdots, n$, the sequence $\left\{g_{k}^{(i)}(t)\right\}$ converges in $R$. Therefore, for each $i=0,1,2, \cdots, n$, there exists $G_{i}(t) \in D^{n}\left[t_{0}-\lambda, T\right]$ such that

$$
\lim _{k \rightarrow \infty} g_{k}^{(i)}(t)=G_{i}(t)
$$

Since $\ell(t)$ is bounded on $\left[t_{0}-\lambda, T\right]$ and $\left\{g_{k}^{(i)}(t)\right\}$ converges uniformly to $G_{i}(t)$ for each $i=0,1,2, \ldots, n$, so $G_{i}(t), i=0,1,2, \cdots, n$, is continuous and

$$
\mathrm{G}_{\mathfrak{i}}^{\prime}(\mathrm{t})=\mathrm{G}_{\mathrm{i}+1}(\mathrm{t}),
$$

which shows that $G_{i}(t)$ is $n$ times continuously differential and hence $\left(D^{n}\left[t_{0}-\lambda, T\right], d\right)$ is complete.

Next, define an operator $\mathfrak{R}: \mathrm{D}^{\mathfrak{n}}\left[\mathrm{t}_{0}-\lambda, T\right] \rightarrow \mathrm{D}^{\mathfrak{n}}\left[\mathrm{t}_{0}-\lambda, T\right]$ by

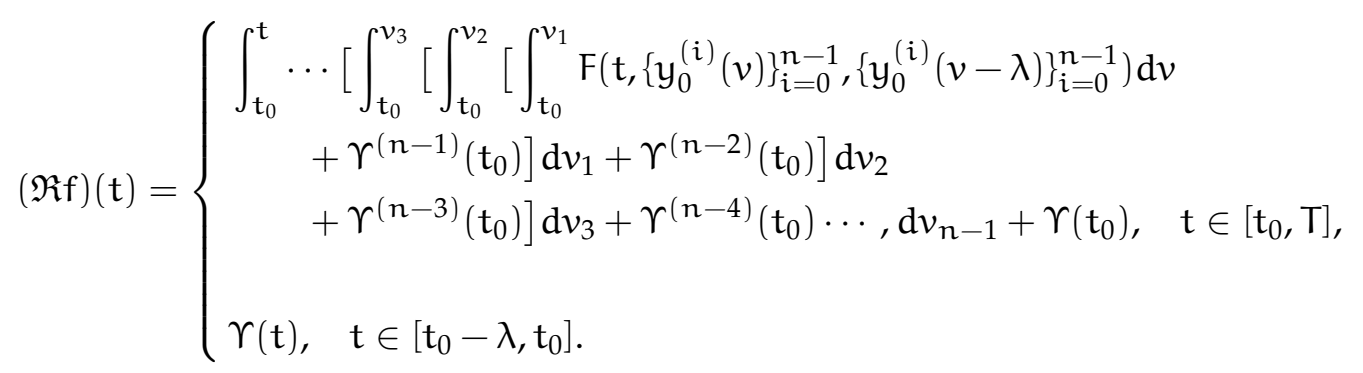

Since $F\left(t,\left\{y_{0}^{(i)}(v)\right\}_{i=0}^{n-1},\left\{y_{0}^{(i)}(v-\lambda)\right\}_{i=0}^{n-1}\right)$ and $\Upsilon(t)$ are continuous on $\left[t_{0}, T\right]$ and $\left[t_{0}-\lambda, t_{0}\right]$ respectively, therefore, $\mathfrak{R}$ is well-defined.

Next we show that the operator is strictly contractive. For this, for any $y_{1}, y_{2} \in X$ let $d\left(y_{1}, y_{2}\right)=C_{y_{1} y_{2}}$, i.e.,

$$
\left|y_{1}^{(i)}(t)-y_{2}^{(i)}(t)\right| \leqslant C_{y_{1} y_{2}} \ell(t),
$$

for all $t \in\left[t_{0}-\lambda, T\right]$ and $i=0,1,2, \cdots, n$. Now

$$
\begin{aligned}
& \left|\left(\mathfrak{R} y_{1}\right)^{(j)}(t)-\left(\mathfrak{R} y_{2}\right)^{(j)}(t)\right|=\mid \int_{t_{0}}^{t} \ldots \int_{t_{0}}^{v_{3}} \int_{t_{0}}^{v_{2}} \int_{t_{0}}^{v_{1}}\left[F\left(t,\left\{y_{1}^{(i)}(v)\right\}_{i=0}^{n-1},\left\{y_{1}^{(i)}(v-\lambda)\right\}_{i=0}^{n-1}\right)\right. \\
& \left.-F\left(t,\left\{y_{2}^{(i)}(v)\right\}_{i=0}^{n-1},\left\{y_{2}^{(i)}(v-\lambda)\right\}_{i=0}^{n-1}\right)\right] d v d v_{1} d v_{2} d v_{3} \cdots d v_{n-1-j} \\
& \leqslant \mid \int_{t_{0}}^{t} \cdots \int_{t_{0}}^{v_{3}} \int_{t_{0}}^{v_{2}} \int_{t_{0}}^{v_{1}}\left[\sum_{i=0}^{n-1} L_{i+1}\left|y_{1}^{(i)}(t)-y_{2}^{(i)}(t)\right|\right.
\end{aligned}
$$




$$
\begin{aligned}
& \left.+\sum_{i=0}^{n-1} L_{n+i+1}\left|y_{1}^{(i)}(t-\lambda)-y_{2}^{(i)}(t-\lambda)\right|\right] d v d v_{1} d v_{2} d v_{3} \cdots d v_{n-1-j} \mid \\
\leqslant & C_{y_{1} y_{2}}\left|\sum_{i=1}^{2 n} L_{i} \int_{t_{0}}^{t} \cdots \int_{t_{0}}^{v_{3}} \int_{t_{0}}^{v_{2}} \int_{t_{0}}^{v_{1}} \ell(t) d v d v_{1} d v_{2} d v_{3} \cdots d v_{n-1-j}\right| \\
\leqslant & K \sum_{i=1}^{2 n} L_{i} C_{y_{1} y_{2}} \ell(t) \\
\leqslant & K \sum_{i=1}^{2 n} L_{i} d\left(y_{1}, y_{2}\right),
\end{aligned}
$$

for all $t \in\left[t_{0}, T\right]$ and $j=0,1,2, \cdots, n$. Also $\left|\left(\mathfrak{R} y_{1}\right)^{(j)}(t)-\left(\mathfrak{R} y_{2}\right)^{(j)}(t)\right|=0$ for all $t \in\left[t_{0}-\lambda, t_{0}\right]$.

Since we have assumed that $0<K \sum_{i=1}^{2 n} L_{i}<1$, therefore the operator is strictly contractive. Moreover, it follows that for any $y_{0} \in X$ there exists $C, 0 \leqslant C<\infty$, such that

$$
\max _{0 \leqslant i \leqslant n}\left|\left(\mathfrak{R} y_{0}\right)^{(i)}(t)-y_{0}^{(i)}(t)\right| \leqslant C \ell(t), t \in\left[t_{0}-\lambda, T\right] .
$$

Since $F\left(t,\left\{y^{(i)}(t)\right\}_{i=0}^{n-1},\left\{y^{(i)}(t-\lambda)\right\}_{i=1}^{n-1}\right)$ and $y_{0}^{(j)}(t), j=0,1,2, \cdots, n$, are bounded on $\left[t_{0}-\lambda, T\right]$ and also $\min _{t \in\left[t_{0}-\lambda, T\right]} \ell(t)>0$, so we have

$$
\mathrm{d}\left(\left(\mathfrak{R} \mathrm{y}_{0}\right)(\mathrm{t}), \mathrm{y}_{0}(\mathrm{t})\right)<\infty .
$$

From Theorem 2.3, we deduce that the sequence $\left\{\mathfrak{R}^{n} x\right\}$ converges to a fixed point $u$ in $X$. Thus,

$$
(\Re u)(t)=u(t),
$$

for all $t \in\left[t_{0}-\lambda, T\right]$. Consequently $u(t)$ is the solution to (1.1).

Next we show that $u(t)$ is the unique such element in $X$. For this we will show that

$$
\{y \in X: d(u, y)<\infty\}=X .
$$

Since for every $y \in X, y^{(i)}(t)$ is bounded on $\left[t_{0}-\lambda, T\right]$ and $\min _{t \in\left[t_{0}-\lambda, T\right]} \ell(t)>0$, so there can be found a constant $\mathrm{C}$ such that $0<\mathrm{C}<\infty$ and

$$
\left|y^{(i)}(t)-u^{(i)}(t)\right| \leqslant C \ell(t)
$$

for all $t \in\left[t_{0}-\lambda, T\right]$ and $i=0,1,2, \cdots, n$, which implies $d(y, u)<\infty$, for all $y \in X$. Hence, $\{y \in X$ : $\mathrm{d}(\mathrm{y}, \mathrm{u})<\infty\}=X$ and therefore $u$ is unique in $\mathrm{X}$. Now we derive from the assumptions that

$$
-\ell(t) \leqslant y^{(n)}(t)-F\left(t,\left\{y^{(i)}(t)\right\}_{i=0}^{n-1},\left\{y^{(i)}(t-\lambda)\right\}_{i=0}^{n-1}\right) \leqslant \ell(t), \quad t \in\left[t_{0}, T\right],
$$

and

$$
\left|y^{(i)}(t)-\Upsilon^{(i)}(t)\right| \leqslant \ell(t), \quad i=0,1, \cdots, n-1, \quad t \in\left[t_{0}-\lambda, t_{0}\right] .
$$

Integrating $n$ times each term in the first inequality from $t_{0}$ to $t$, with adding and subtracting $\Upsilon^{(n-i)}(t)$ with each $i$-th time integration we get the following

$$
\begin{aligned}
|y(t)-(\Re y)(t)| \leqslant & K \ell(t)+\sum_{i=1}^{n-1} \int_{t_{0}}^{t} \ldots \int_{t_{0}}^{v_{3}} \int_{t_{0}}^{v_{2}} \int_{t_{0}}^{v_{1}}\left|\Upsilon^{(n-i)}\left(t_{0}\right)-y^{(n-i)}\left(t_{0}\right)\right| d v_{0} d v_{1} d v_{2} d v_{3} \cdots d v_{n-1-i} \\
& +\left|\Upsilon\left(t_{0}\right)-y\left(t_{0}\right)\right|,
\end{aligned}
$$

for all $t \in\left[t_{0}-\lambda, T\right]$. Using the second inequality above we have

$$
|y(t)-(\Re y)(t)| \leqslant(n K+1) \ell(t), \quad t \in\left[t_{0}-\lambda, T\right] .
$$


Therefore,

$$
d(y(t),(\Re y)(t)) \leqslant(n K+1)
$$

From Theorem 2.3 it follows that

$$
d(y, u) \leqslant \frac{1}{1-K \sum_{i=1}^{2 n} L_{i}} d((\Re y)(t), y(t)) \leqslant \frac{n K+1}{1-K \sum_{i=1}^{2 n} L_{i}} .
$$

This completes the proof.

\section{Hyers-Ulam stability}

In the following theorem, we state without a proof the Hyers-Ulam stability of (1.1) on $\left[t_{0}-\lambda, T\right]$. The proof can be done with the same steps as that of the theorem above, but with a slight change in the metric, i.e., the proof needs the metric defined below

$$
\mathrm{d}\left(\mathrm{y}_{1}, \mathrm{y}_{2}\right)=\inf \left\{\mathrm{C} \in[0, \infty]: \max _{0 \leqslant i \leqslant n}\left|\mathrm{y}_{1}^{(i)}(\mathrm{t})-\mathrm{y}_{2}^{(i)}(\mathrm{t})\right| \leqslant C, \quad \forall \mathrm{t} \in\left[\mathrm{t}_{0}-\lambda, \mathrm{T}\right]\right\} .
$$

Theorem 4.1. Let $L$ be the length of the interval $\left[\mathrm{t}_{0}, \mathrm{~T}\right]$ and $\mathrm{L}_{1}, \mathrm{~L}_{2}, \mathrm{~L}_{3}, \cdots, \mathrm{L}_{2} \mathrm{n}$ be positive constants such that $0<\mathrm{L}^{\mathrm{m}} \sum_{i=1}^{2 n} \mathrm{~L}_{\mathrm{i}}<1$, for all $\mathrm{m}=0,1,2, \cdots, \mathrm{n}$. Let $\mathrm{F}:\left[\mathrm{t}_{0}, \mathrm{~T}\right] \times \mathrm{R}^{2 \mathrm{n}} \rightarrow \mathrm{R}$ be a continuous bounded function with the Lipschitz condition:

$$
\left|F\left(t, x_{1}, x_{3}, \cdots, x_{2 n}\right)-F\left(t, y_{1}, y_{2}, \cdots, y_{2 n}\right)\right|<\sum_{i=1}^{2 n} L_{i}\left|x_{i}-y_{i}\right|,
$$

for all $\mathrm{t} \in\left[\mathrm{t}_{0}, \mathrm{~T}\right]$ and $\mathrm{x}_{\mathrm{i}}, \mathrm{y}_{\mathrm{i}} \in \mathrm{R}$, where $\mathrm{i}=1,2,3, \cdots, 2 \mathrm{n}$. Then equation (1.1) has Hyers-Ulam stability on $\left[t_{0}-\lambda, T\right]$.

\section{Acknowledgment}

The authors express their sincere gratitude to the editors and anonymous referees for the careful reading of the original manuscript and useful comments that helped to improve the presentation of the results and accentuate important details. This work was supported by the National Natural Science Foundation of China (11571378).

\section{References}

[1] T. Aoki, On the stability of the linear transformation in Banach spaces, J. Math. Soc. Japan, 2 (1950), 64-66. 1

[2] G.-Y. Choi, S.-M. Jung, Invariance of Hyers-Ulam stability of linear differential equations and its applications, Adv. Difference Equ., 2015 (2015), 14 pages. 1

[3] J. B. Diaz, B. Margolis, A fixed point theorem of the alternative, for contractions on a generalized complete metric space, Bull. Amer. Math. Soc., 74 (1968), 305-309. 2

[4] J.-H. Huang, Q. H. Alqifiary, Y.-J. Li, Superstability of differential equations with boundary conditions, Electron. J. Differential Equations, 2014 (2014), 8 pages. 1

[5] J.-H. Huang, S.-M. Jung, Y.-J. Li, On Hyers-Ulam stability of nonlinear differential equations, Bull. Korean Math. Soc., 52 (2015), 685-697. 1

[6] J.-H. Huang, Y.-J. Li, Hyers-Ulam stability of linear functional differential equations, J. Math. Anal. Appl., 426 (2015), 1192-1200. 1

[7] J.-H. Huang, Y.-J. Li, Hyers-Ulam stability of delay differential equations of first order, Math. Nachr., 289 (2016), 60-66. 1

[8] D. H. Hyers, On the stability of the linear functional equation, Proc. Nat. Acad. Sci. U. S. A., 27 (1941), 222-224. 1

[9] S.-M. Jung, Hyers-Ulam stability of linear differential equations of first order, Appl. Math. Lett., 17 (2004), 1135-1140. 1

[10] S.-M. Jung, A fixed point approach to the stability of differential equations $y^{\prime}=F(x, y)$, Bull. Malays. Math. Sci. Soc., 33 (2010), 47-56. 1 
[11] S.-M. Jung, J. Brzdęk, Hyers-Ulam stability of the delay equation $y^{\prime}(t)=\lambda y(t-\tau)$, Abstr. Appl. Anal., 2010 (2010), 10 pages. 1

[12] S.-M. Jung, J. Roh, The linear differential equations with complex constant coefficients and Schr'odinger equations, Appl. Math. Lett., 66 (2016), 6 pages. 1

[13] Y. Kuang, Delay differential equations with applications in population dynamics, Mathematics in Science and Engineering, Academic Press, Inc., Boston, MA, (1993). 1

[14] Y.-J. Li, Y. Shen, Hyers-Ulam stability of nonhomogeneous linear differential equations of second order, Int. J. Math. Math. Sci., 2009 (2009), 7 pages. 1

[15] T.-X. Li, A. Zada, Connections between Hyers-Ulam stability and uniform exponential stability of discrete evolution families of bounded linear operators over Banach spaces, Adv. Difference Equ., 2016 (2016), 8 pages. 1

[16] T. Miura, S. Miyajima, S.-E. Takahasi, A characterization of Hyers-Ulam stability of first order linear differential operators, J. Math. Anal. Appl., 286 (2003), 136-146. 1

[17] Z. Moszner, Stability has many names, Aequationes Math., 90 (2016), 983-999. 1

[18] M. Obloza, Hyers stability of the linear differential equation, Rocznik Nauk.-Dydakt. Prace Mat., 13 (1993), 259-270. 1

[19] M. Obloza, Connections between Hyers and Lyapunov stability of the ordinary differential equations, Rocznik Nauk.Dydakt. Prace Mat., 14 (1997), 141-146. 1

[20] D. Otrocol, V. Ilea, Ulam stability for a delay differential equation, Cent. Eur. J. Math., 11 (2013), 1296-1303. 1

[21] T. M. Rassias, On the stability of the linear mapping in Banach spaces, Proc. Amer. Math. Soc., 72 (1978), 297-300. 1

[22] C. Tunç, E. Biçer, Hyers-Ulam-Rassias stability for a first order functional differential equation, J. Math. Fundam. Sci., 47 (2015), 143-153. 1

[23] S. M. Ulam, A collection of mathematical problems, Interscience Tracts in Pure and Applied Mathematics, Interscience Publishers, New York-London, (1960). 1

[24] J.-R. Wang, M. Feĉkan, Y. Zhou, On the stability of first order impulsive evolution equations, Opuscula Math., 34 (2014), 639-657. 1

[25] B. Xu, J. Brzdęk, W.-N. Zhang, Fixed point results and the Hyers-Ulam stability of linear equations of higher orders, Pacific J. Math., 273 (2015), 483-498.

[26] A. Zada, S. Faisal, Y.-J. Li, On the Hyers-Ulam stability of first-order impulsive delay differential equations, J. Funct. Spaces, 2016 (2016), 6 pages.

[27] A. Zada, O. Shah, R. Shah, Hyers-Ulam stability of non-autonomous systems in terms of boundedness of Cauchy problems, Appl. Math. Comput., 271 (2015), 512-518. 1 